\title{
Age trajectories of physiological indices in relation to healthy life course
}

\author{
Konstantin G. Arbeev*, Svetlana V. Ukraintseva, Igor Akushevich, Alexander M. Kulminski, \\ Liubov S. Arbeeva, Lucy Akushevich, Irina V. Culminskaya, Anatoliy I. Yashin
}

Centre for Population Health and Aging, Duke University, USA

\section{A R T I C L E I N F O}

\section{Article history:}

Received 17 May 2010

Received in revised form 22 December 2010

Accepted 16 January 2011

Available online 22 January 2011

\section{Keywords:}

Stress resistance

Adaptive capacity

Age-dynamics

Physiological norm

Framingham Heart Study

\begin{abstract}
A B S T R A C T
We analysed relationship between the risk of onset of "unhealthy life" (defined as the onset of cancer, cardiovascular diseases, or diabetes) and longitudinal changes in body mass index, diastolic blood pressure, hematocrit, pulse pressure, pulse rate, and serum cholesterol in the Framingham Heart Study (Original Cohort) using the stochastic process model of human mortality and aging. The analyses demonstrate how decline in resistance to stresses and adaptive capacity accompanying human aging can be evaluated from longitudinal data. We showed how these components of the aging process, as well as deviation of the trajectories of physiological indices from those minimising the risk at respective ages, can lead to an increase in the risk of onset of unhealthy life with age. The results indicate the presence of substantial gender difference in aging related decline in stress resistance and adaptive capacity, which can contribute to differences in the shape of the sex-specific patterns of incidence rates of aging related diseases.
\end{abstract}

(c) 2011 Elsevier Ireland Ltd. All rights reserved.

\section{Introduction}

Individual measurements of various physiological indices in humans represent "snapshots" of individual's physiological state at particular ages. Longitudinal data on aging, health, and longevity describe individual age trajectories of such indices in connection with changes in health and survival status. Such data contain important information not only about values of physiological state at a given age, but also about dynamic characteristics of specific indices, which, together with values, may be associated with morbidity and mortality risks. In our earlier studies we showed that dynamic characteristics of blood glucose, body mass index, diastolic blood pressure, hematocrit, pulse pressure, pulse rate and total cholesterol at middle and old ages are related to risks of death and onset of cancer, cardiovascular diseases (CVD) and diabetes at later ages (Yashin et al., 2006, 2010a). Note that many associations between selected characteristics and respective risks could be evaluated using standard statistical approaches. Such evaluations are useful at the initial step of analyses when the presence of specific effects has to be identified. However, for studying mechanisms driving aging related changes such methods are not appropriate, because they ignore existing knowledge about regularities of such changes, and do not allow for linking new

\footnotetext{
* Corresponding author at: Centre for Population Health and Aging, Duke University, Department of Sociology, Trent Hall, 331 Trent Drive, Room 002, Box 90408, Durham, NC 27708-0408, USA. Tel.: +1 919668 2707; fax: +1 9196843861.

E-mail address: konstantin.arbeev@duke.edu (K.G. Arbeev).
}

findings with available information and concepts of aging available in the literature.

In this paper we use such knowledge about several major concepts of aging to link observed outcomes (morbidity/mortality risks, or longevity/healthy longevity) and longitudinal measurements of physiological indices. This allows for constructing dynamic models describing mechanisms of aging related changes involved in generating longitudinal data in connection with morbidity/mortality risks. Respective mechanisms, as well as characteristics of the risks, can be evaluated from longitudinal data by applying appropriate statistical modeling techniques (Yashin et al., 2007a). This approach has been applied to analyzing data on longitudinal measurements of blood glucose (BG) in the Framingham heart study (FHS). It allowed for investigating dynamic mechanisms that relate age trajectories of BG and chances of reaching exceptional longevity. It also allowed for separating effects of persistent external disturbances (effects of allostatic adaptation) from "normal" aging-related changes developing in human body due to the senescence process (Yashin et al., 2009, 2010b).

In this paper we apply this approach to investigate mechanisms linking age trajectories of physiological indices and risks of onset of aging-related diseases. For this purpose, we apply the model by Yashin et al. (2007a) to analyse relationship between the risk of onset of "unhealthy life" (defined as onset of cancer, CVD, or diabetes) and longitudinal changes in six physiological indices (body mass index, diastolic blood pressure, hematocrit, pulse pressure, pulse rate, and serum cholesterol) whose dynamic characteristics have been shown to be related to the risk of onset of 
"unhealthy life" in participants of the original cohort of the Framingham Heart Study (Yashin et al., 2010a). We show how different components of the aging process, such as the decline in resistance to stresses and adaptive capacity, can be evaluated from age trajectories of these indices and data on onset of diseases, and how they can contribute to an increase in the risk of onset of "unhealthy life" with age. We calculate respective characteristics in females and males and evaluate their contribution to the observed sex-specific patterns of incidence rate of onset of "unhealthy life."

\section{Data and method}

\subsection{Framingham Heart study (FHS) data}

The FHS Original Cohort consists of 5209 respondents (nearly all are Caucasians, $46 \%$ male) aged 28-62 years at baseline and residing in Framingham, Massachusetts, between 1948 and 1951, and who had not yet developed overt symptoms of cardiovascular disease or suffered a heart attack or stroke (Dawber, 1980; Dawber et al., 1951). The study continues to the present with biennial examinations (29 exams to date, data from exams 1-25 were used in this study) that include detailed medical history, physical exams, and laboratory tests. The FHS Original Cohort has been followed for about 60 years for the occurrence of cardiovascular diseases, cancer, diabetes mellitus, and death. Examination of participants, including an interview, physical examination, and laboratory tests, has been taken biennially. Phenotypic traits collected in the FHS Original Cohort over 60 years and relevant to our analyses include: life span, ages at onset of diseases (with the emphasis on cardiovascular diseases, cancer, and diabetes mellitus), as well as indices characterising physiological state. The occurrence of diseases (CVD and cancer) and death has been followed through continuous surveillance of hospital admissions, death registries, clinical exams, and other sources, so that all the respective events are included in the study. We used data on first occurrence of CVD (defined by the FHS investigators as having any of the following: coronary heart disease, intermittent claudication, congestive heart failure, or stroke/transient ischemic attack) and cancer from the follow-up data, and data on current diabetes status (defined by the FHS investigators as a level of blood glucose exceeding $140 \mathrm{mg} / \mathrm{dl}$ and/or taking insulin or oral hypoglycemics) in exams to calculate the age at onset of "unhealthy life" as the minimum of ages of occurrence of these diseases. If an individual did not contract any of these diseases during the observation period, then that individual was considered censored at the age of the last follow-up or death. Individuals who had any of the diseases before (for cancer and CVD) or at (for diabetes) the first FHS exam were excluded from the analyses of "unhealthy life." Data on physiological indices that we used include: body mass index (BMI, exams 1-25), diastolic blood pressure (DBP, exams 1-25), hematocrit (HCT, exams 4-21), pulse pressure (PP, exams 1-25), pulse rate (PR, exams 1,4-25), and serum cholesterol (SCH, exams 1-11, 13-15, 20, 22-25).

\subsection{The underlying paradigm linking age dynamics of physiological indices and morbidity risks}

The paradigm linking the age dynamics of physiological indices and morbidity risks is based on several major concepts of aging known to date that are incorporated in the framework of the model by Yashin et al. (2007a).

The concept of allostasis describes the adaptive response of an organism to the persistent environmental and psychosocial situations, commonly referred to as "stresses" (Sterling and Eyer, 1988). Allostasis means that an organism maintains "stability" of some functions by changing the others. Unlike homeostasis, allostasis refers to the organism's ability to cope physiologically, behaviorally, and emotionally with specific environmental challenges while maintaining the regulatory control of the homeostatic systems that operate within narrow parameters (McEwen and Wingfield, 2003). The body pays the "price" (allostatic load) for being forced to adapt to persistent environmental and psychosocial challenges. The allostatic load depends on how inefficient defense and compensatory mechanisms are, and/or how many stressful challenges an individual experienced. Over time, allostatic load can accumulate and the overexposure to neural, endocrine, and immune stress mediators can have adverse effects on various organ systems leading to the development of a disease (and, eventually, increasing mortality risk). Thus, investigation of effects of allostatic adaptation provides us with important indicators of physiological pre-disease pathways. Several practical procedures for evaluating allostatic load were suggested (Seeman et al., 1997, 2001). These procedures, however, do not take into account the dynamic nature of physiological processes affecting individual health and survival. Important information for understanding dynamic mechanisms regulating age trajectories of physiological state in individuals is that persistent exposure to external disturbances during the life course modifies set points of biological homeostatic regulation resulting in shifting individual's physiological state from its "optimal" age trajectory where mortality risk is minimal. Thus the effects of allostatic adaptation are reflected in the individual age trajectories of physiological indices, and, therefore, they need to be included in the mathematical model describing age dynamics of these indices.

The decline in adaptive (homeostatic) capacity of physiological and biological systems with age ("homeostenosis") has been shown to be an important characteristic of aging (Hall et al., 2000; Rankin and Kushner, 2009; Troncale, 1996) and, hence, it needs to be accounted for in description of the model.

In analyses of effects of persistent environmental and psychosocial challenges (which we name "stresses") on an organism's functioning one should take into account that available longitudinal studies (including the FHS) typically contain scarce (if any) information on external disturbances affecting individuals during their life course. The intensities (and magnitudes) of persistent external stresses that affect an organism's functioning are generally unknown. Therefore, the direct estimation of such "stresses" from the data is not possible. Our approach was developed to make possible estimation of the effects of allostatic adaptation from longitudinal data. It includes effects of allostatic adaptation into the model describing age trajectories of physiological state. As we show, these trajectories can be evaluated from the data, along with the negative feedback coefficient. The specific "stresses" causing this dynamics remain, nevertheless, unknown and unobserved.

Taken together, these considerations can be formalised in the following stochastic differential equation describing the age dynamics of a physiological index with age (Yashin et al., 2007a):

$d Y_{t}=a(t)\left(Y_{t}-f_{1}(t)\right) d t+b(t) d W_{t}, Y_{0}$

Here $Y_{t}$ is the value of a physiological index at age $t$. The function $f_{1}(t)$ describes the effect of allostatic adaptation, i.e., the trajectory that individual's physiological index is forced to follow by homeostatic forces in the presence of external disturbances described by a Wiener process $W_{t}$ (which is independent of the initial normally distributed value $Y_{0}$ ). The strength of homeostatic forces is characterised by the negative feedback coefficient $a(t)$ : larger values of this function correspond to faster return of the trajectory of the physiological index to the allostatically prescribed values $f_{1}(t)$. Therefore, the decline in the absolute value of this function with age represents the effect of homeostenosis. In our applications to data on onset of "unhealthy life," we used a linear approximation of such decline: $a(t)=a_{Y}+b_{Y} t$ (with $a_{Y}<0$ and $b_{Y}<0$ ). We used the quadratic function to model the "allostatic trajectory" $f_{1}(t)$ (which we denote the "mean allostatic state"): $f_{1}(t)=a_{f_{1}}+b_{f_{1}} t+c_{f_{1}} t^{2}$. The choice of the quadratic function for the "mean allostatic state" comes from the empirical observations of the average trajectories of the physiological indices in the FHS, which generally have a quadratic form (see Fig. 1), although, of course, the mean trajectories does not necessary have to follow those of $f_{1}(t)$ exactly.

The concept of allostatic adaptation implicitly assumes the notion of "deviation from the norm," that is, the "normal" state of an organism corresponding to "optimal" functioning in terms of minimising morbidity or mortality risk. The studies of how persistent external unfavourable conditions get "under the skin" of affected person increasing his/her susceptibility to diseases and death (McEwen, 2000; Sterling and Eyer, 1988) provide evidence that many such conditions affect set-points of physiological homeostasis changing physiological balance from the "normal" to "abnormal" state. This means that the trajectory of a physiological index that an organism is forced to follow under the persistent external disturbances $\left(f_{1}(t)\right)$ may be different from the "optimal" trajectory (which we denote $f(t)$ ) minimising mortality/morbidity risk that the homeostatic regulation would force to follow in the absence of external disturbances. The difference between $f_{1}(t)$ and such "optimal" trajectory (which can be interpreted as agespecific "physiological norm") provides the measure of the allostatic load. In our applications to the data on onset of "unhealthy life," we used a linear approximation of the "optimal" trajectory: $f(t)=a_{f}+b_{f} t$ (see also "Sensitivity Analyses" in Appendix A).

Introduction of the notion of "optimal" age trajectory of physiological index allows for linking longitudinal measurements of physiological indices and morbidity (or mortality) risk. As assumed in the definition of the "optimal" age trajectory $f(t)$, deviations of physiological index from respective "optimal" levels for each age (represented by this function $f(t)$ ) increase individual's chances to develop a disease or die. Different studies observed U- or J- shape of the risks as functions of various physiological indices (Allison et al., 1997; Boutitie et al., 2002; Kulminski et al., 2008a; Kuzuya et al., 2008; Mazza et al., 2007; Okumiya et al., 1999 Protogerou et al., 2007; Troiano et al., 1996; van Uffelen et al., 2010; Witteman et al., 1994; Yashin et al., 2001). Following these observations, we suggested using a quadratic function to model dependence of the risk on deviations of trajectories of physiological index $Y_{t}$ from the optimal trajectory $f(t)$ (Arbeev et al., 2009; Yashin et al., 2007c, 2008, 2009, 2010b), although other functional forms can be used as well (Yashin et al., 2007b). We use the following expression for the hazard rate (i.e., incidence rate for onset of "unhealthy life" in our applications) using the onedimensional version of the model by Yashin et al. (2007a):

$\mu\left(t, Y_{t}\right)=\mu_{0}(t)+\left(Y_{t}-f(t)\right)^{2} Q(t)$

here $\mu_{0}(t)$ is the "residual" or "baseline" hazard that represents the risk which would remain if the index $Y_{t}$ followed the "optimal trajectory" represented by the function $f(t)$. It models the effect of other factors (such as the senescence process) 
body mass index

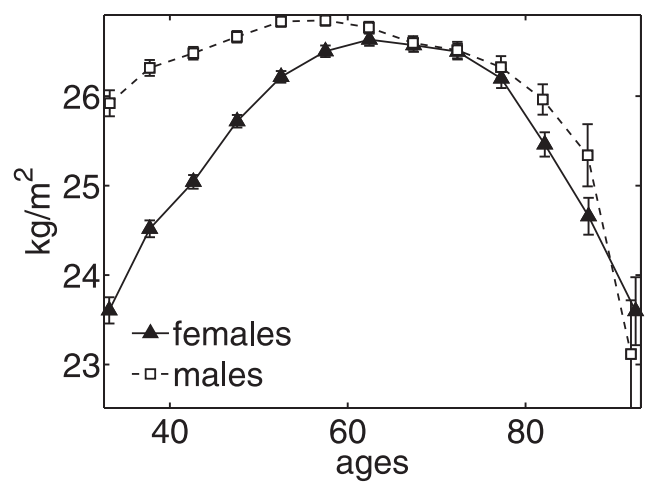

hematocrit

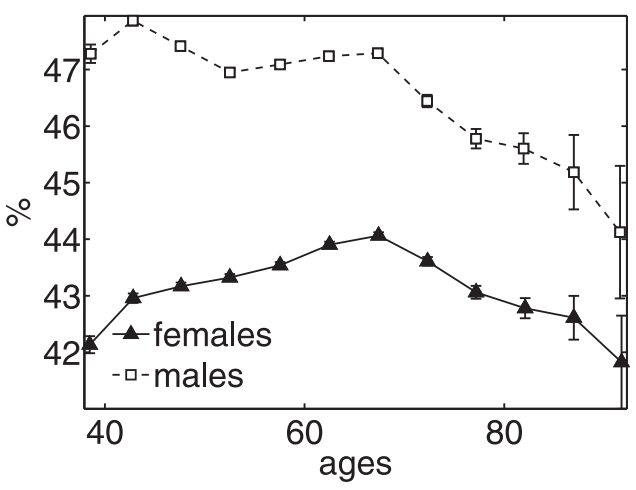

pulse rate

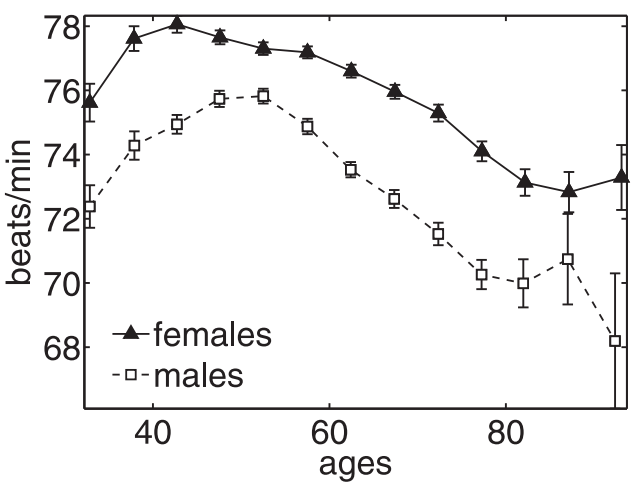

diastolic blood pressure

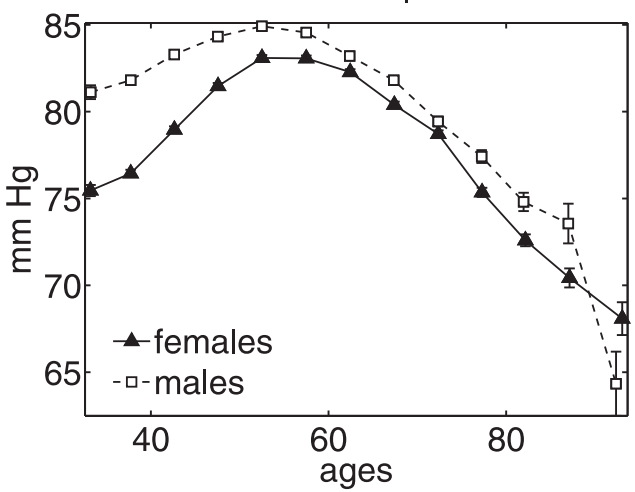

pulse pressure

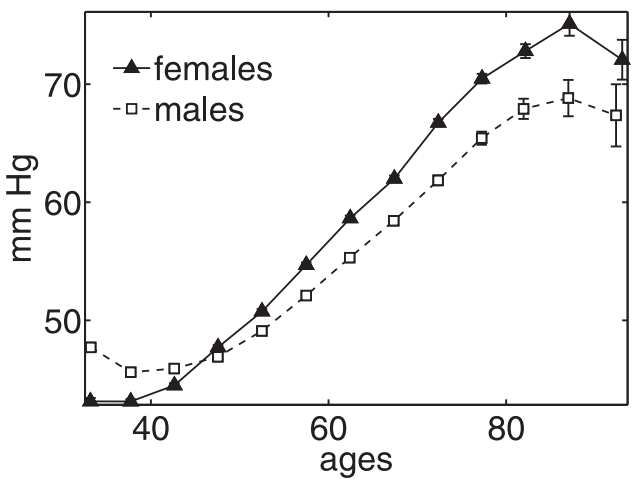

serum cholesterol

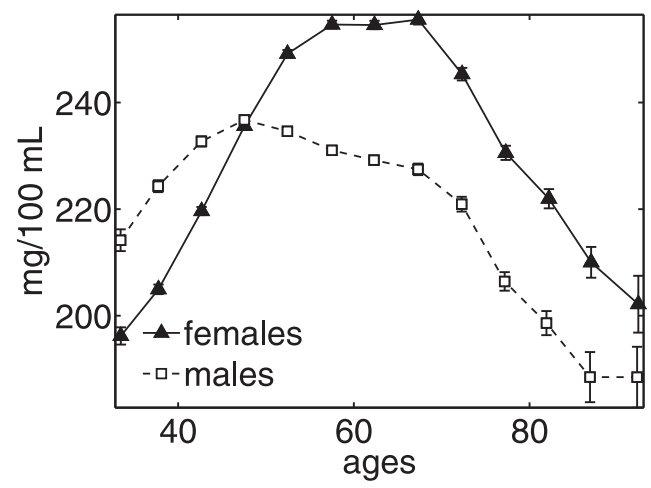

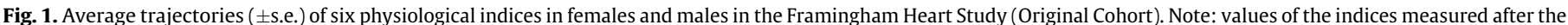
onset of any of the three diseases defining "unhealthy life" (i.e., cancer, CVD, diabetes) are excluded from the calculations.

that impact the incidence rate. In applications to data on onset of "unhealthy life," we specified the logistic (gamma-Gompertz) function for the baseline hazard:

$\mu_{0}(t)=\frac{\mu_{0}^{0}(t)}{1+\sigma_{2}^{2} \int_{0}^{t} \mu_{0}^{0}(u) d u}$

where $\mu_{0}^{0}(t)=a_{\mu_{0}} e^{b_{\mu_{0}} t}$. The specific choice for the baseline hazard is motivated by the earlier observations that the incidence rates of many aging-related diseases (including cancer, heart disease, stroke and diabetes, which are used in the definition of onset of "unhealthy life" in our study) decelerate or even decline at advanced ages (Arbeev et al., 2005; Rockwood et al., 2000; Ukraintseva et al., 2006, 2010; Ukraintseva and Yashin, 2001, 2003), and by empirical observations of the incidence rates of onset of "unhealthy life" in the present study (Fig. 2)

The non-negative function $Q(t)$ characterises sensitivity of the risk function (incidence rate) to deviations of physiological variables from the "optimal" function $f(t)$. This function can be interpreted as the "vulnerability" index. It characterises the "robustness", or "vulnerability," component of stress resistance. When the value of this function increases (i.e., the U-shape of the risk narrows), an organism becomes more vulnerable to deviations from the "normal" state caused by external disturbances (because the same magnitude of deviation from the "optimal" trajectory results in a larger increase in the risk). An increase in this index with age (that is, the decline in stress resistance) can be considered as a manifestation of the senescence process (Strehler, 1962; Strehler and Mildvan, 1960; Ukraintseva and Yashin, 2003). In this study, we model the "vulnerability" index as a linear function of age: $Q(t)=a_{Q}+b_{Q} t$ (see also "Sensitivity Analyses" in Appendix A).

Fig. 3 schematically illustrates the main features of the model. A discrete-time version of model (1)-(2) (with values of physiological indices evaluated at one-year age intervals using respective observations in the adjacent FHS exams) was applied to data on onset of "unhealthy life" in females and males in the FHS Original Cohort. Parameters of adaptive capacity, mean allostatic state, the vulnerability index, the baseline hazard and the "optimal" trajectory were specified as described above. The diffusion coefficient $b(t)$ was modelled constant. We also performed several sensitivity analyses to test alternative specifications of the model (see "Sensitivity Analyses" in Appendix A)

\section{Results and discussion}

Estimates of parameters of the baseline incidence rate, $\mu_{0}(t)$, the "vulnerability" index, $Q(t)$, the age-specific adaptive capacity $a(t)$, the "mean allostatic state" $f_{1}(t)$, and the age-specific "norm" 


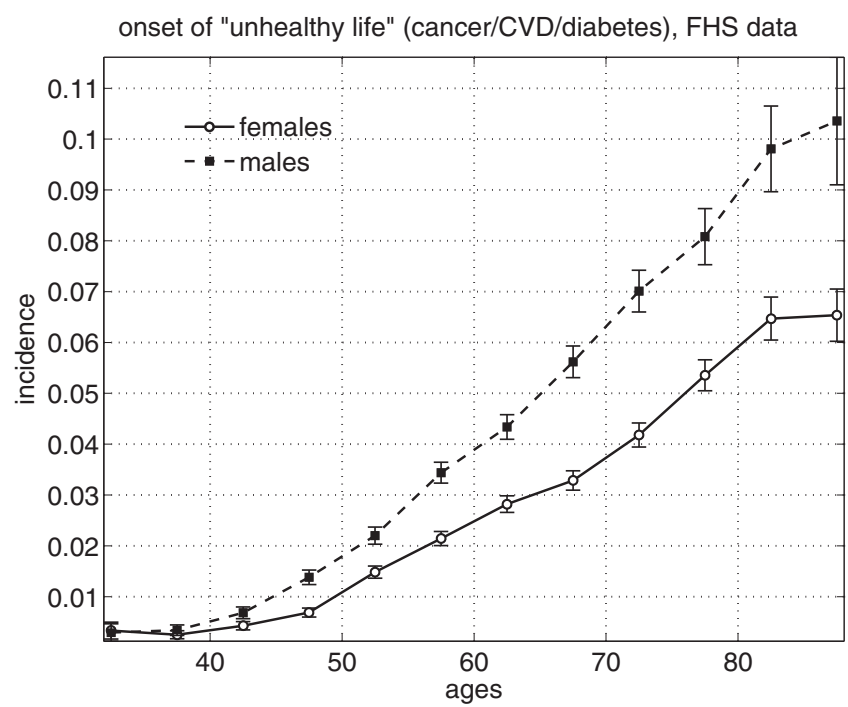

Fig. 2. Incidence rates ( \pm s.e.) of onset of "unhealthy life" (i.e., cancer, CVD, or diabetes) in females and males in the Framingham Heart Study (original cohort).

$f(t)$ for six physiological indices in the quadratic hazard model applied to data on onset of "unhealthy life" in females and males in the Framingham heart study (Original Cohort) are given in Table 1.

\subsection{Patterns of baseline incidence rate}

Fig. 4 shows that for all indices and for both sexes the pattern of the baseline incidence rate decelerates with age (respective estimates of parameter $\sigma_{2}$ are non-zero, $p<0.0001$ ). This pattern contributes to the observed shape of the incidence rate at old ages (Fig. 2). Thus, our a priori expectations about the pattern of the risk of onset of "unhealthy life" corresponding to unspecified factors represented by $\mu_{0}(t)$ are confirmed. Earlier we suggested that the decelerated patterns of incidence rates observed for many chronic diseases at old ages may reflect the contribution of basal process of aging in the body which is manifested in slowdown of metabolism,

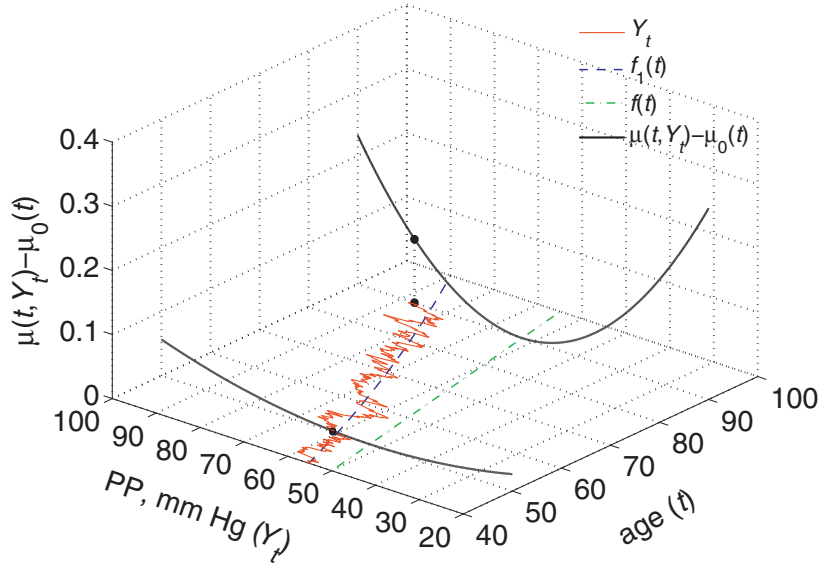

Fig. 3. Illustration of the quadratic hazard model for a hypothetical physiological index $Y_{t}$ (mimicking pulse pressure, $\mathrm{PP}$ ): increase of the hazard rate compared to the baseline level (i.e. $\left.\mu\left(t, Y_{t}\right)-\mu_{0}(t)=\left(Y_{t}-f(t)\right)^{2} Q(t)\right)$ for ages 50 and 90 . Compared to age 50 , individuals at age 90 tend to have a larger increase in the risk (denoted by the dotted line) as the result of larger deviations of the trajectory of the index $\left(Y_{t}\right.$, thin solid line) from the "optimal" trajectory $(f(t)$, dash-dotted line) caused by the divergent trajectory of the "mean allostatic state" $\left(f_{1}(t)\right.$, dashed line) that an organism is forced to follow by the process of adaptive regulation, and a narrower $\mathrm{U}$-shape of the $\operatorname{risk}\left(\mu\left(t, Y_{t}\right)-\mu_{0}(t)\right.$, thick solid line).

proliferative response and information processing with age (Ukraintseva and Yashin, 2001, 2003).

\subsection{Shape of "Vulnerability" index and stress resistance}

The trajectories of the "vulnerability" index $(Q(t))$ increase with age for all indices and both sexes as Fig. 5 illustrates ( $p$-values for the null hypothesis about the constant $Q(t)$ are rejected at different significance levels in all cases except for PP in females and males and $\mathrm{SCH}$ in females, see Table 1). This means that the width of the U-shape of the risk (as a function of respective physiological index) is getting narrower with age. This, in turn, indicates that the range of the values of respective physiological indices corresponding to a "tolerable" increase in the risk is also getting narrower with age

Table 1

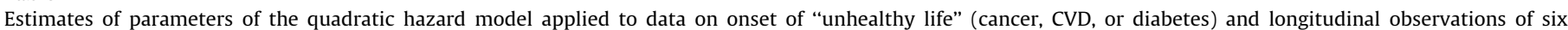
physiological indices in females and males in the Framingham Heart Study (Original Cohort).

\begin{tabular}{|c|c|c|c|c|c|c|c|c|c|c|c|c|c|}
\hline \multirow[t]{2}{*}{ Sex } & \multirow[t]{2}{*}{ Index } & \multicolumn{3}{|c|}{$\begin{array}{l}\text { Baseline hazard } \\
\text { Rate }\left(\mu_{0}(t)\right)\end{array}$} & \multicolumn{2}{|c|}{$\begin{array}{l}\text { Vulnerability } \\
\text { Index }(Q(t))\end{array}$} & \multicolumn{2}{|c|}{$\begin{array}{l}\text { Adaptive } \\
\text { Capacity }(a(t))\end{array}$} & \multicolumn{3}{|c|}{$\begin{array}{l}\text { Allostatic } \\
\text { Trajectory }\left(f_{1}(t)\right)\end{array}$} & \multicolumn{2}{|c|}{$\begin{array}{l}\text { “Optimal” } \\
\text { Trajectory }(f(t))\end{array}$} \\
\hline & & $a_{\mu_{0}} \times 10^{4}$ & $b_{\mu_{0}}$ & $\sigma_{2}$ & $a_{Q} \times 10^{4}$ & $b_{Q} \times 10^{6}$ & $a_{Y}$ & $b_{Y} \times 10^{4}$ & $a_{f_{1}}$ & $b_{f_{1}}$ & $c_{f_{1}}$ & $a_{f}$ & $b_{f}$ \\
\hline \multirow[t]{6}{*}{ Females } & BMI & 0.546 & 0.106 & $1.304^{\dagger}$ & $-0.903^{\#}$ & 3.224 & $-0.017^{\dagger}$ & 1.575 & $9.59^{\dagger}$ & 0.595 & -0.005 & $29.83^{*}$ & -0.113 \\
\hline & DBP & 0.666 & 0.100 & $1.219^{\dagger}$ & $-0.201^{\dagger}$ & 0.716 & $-0.066^{*}$ & 2.357 & $53.69^{\dagger}$ & 1.101 & -0.010 & $93.01^{\S}$ & -0.362 \\
\hline & HCT & 0.044 & 0.146 & $1.632^{\dagger}$ & $-2.715^{\dagger}$ & 9.697 & $-0.089^{\dagger}$ & 7.646 & $33.69^{\dagger}$ & 0.371 & -0.003 & 43.13 & -0.011 \\
\hline & $\mathrm{PP}$ & 0.447 & 0.110 & $1.442^{\dagger}$ & 0.077 & 0.045 & $-0.114^{\dagger}$ & 9.353 & $5.83^{\dagger}$ & 1.054 & -0.001 & 39.72 & 0.028 \\
\hline & PR & 0.515 & 0.107 & $1.268^{\dagger}$ & $-0.131^{\#}$ & 0.466 & $-0.079^{\S}$ & 3.533 & $66.79^{\dagger}$ & 0.490 & -0.005 & 73.90 & -0.078 \\
\hline & $\mathrm{SCH}$ & 0.372 & 0.113 & $1.299^{\dagger}$ & -0.007 & 0.026 & $-0.079^{\dagger}$ & 7.509 & $46.83^{\dagger}$ & 6.827 & -0.056 & 229.28 & -0.161 \\
\hline \multirow[t]{6}{*}{ Males } & BMI & 0.693 & 0.111 & $1.051^{\dagger}$ & $-1.159^{*}$ & 4.814 & $-0.018^{\#}$ & 1.672 & $20.47^{\dagger}$ & 0.254 & -0.003 & 26.09 & -0.057 \\
\hline & DBP & 0.615 & 0.110 & $0.995^{\dagger}$ & $-0.317^{\#}$ & 1.130 & $-0.091^{\dagger}$ & 6.528 & $63.95^{\dagger}$ & 0.878 & -0.009 & 74.87 & -0.017 \\
\hline & HCT & 0.088 & 0.141 & $1.268^{\dagger}$ & $-1.639^{\#}$ & 5.854 & -0.051 & 1.444 & $42.52^{\dagger}$ & 0.224 & -0.003 & $48.80^{\circ}$ & -0.113 \\
\hline & $\mathrm{PP}$ & 0.839 & 0.108 & $1.075^{\dagger}$ & -0.243 & 0.867 & $-0.097^{\dagger}$ & 6.935 & $35.45^{\dagger}$ & 0.058 & 0.006 & 22.64 & 0.393 \\
\hline & PR & 0.686 & 0.110 & $1.044^{\dagger}$ & $-0.178^{\dagger}$ & 0.635 & $-0.074^{*}$ & 3.528 & $66.30^{\S}$ & 0.393 & -0.004 & 70.97 & -0.162 \\
\hline & $\mathrm{SCH}$ & 0.344 & 0.122 & $1.097^{\dagger}$ & $-0.009^{\#}$ & 0.032 & $-0.080^{\dagger}$ & 7.192 & $209.11^{\dagger}$ & 1.235 & -0.017 & 178.36 & -0.270 \\
\hline
\end{tabular}

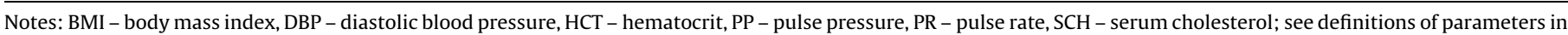
Section 2 .

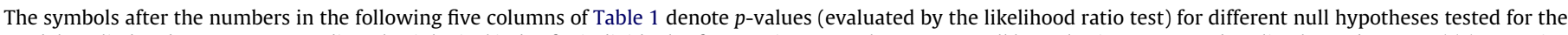

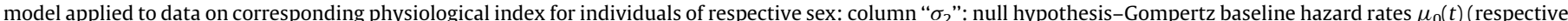

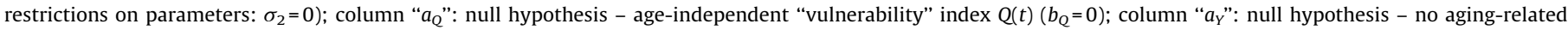

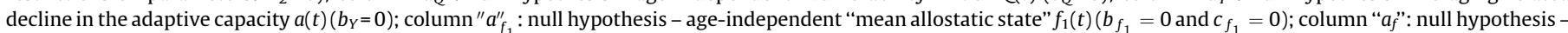

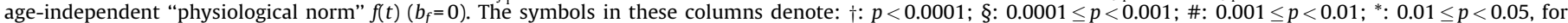

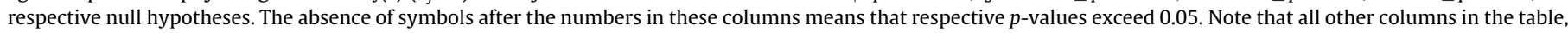
except the five columns mentioned above, are not used to represent information on testing any null hypotheses and therefore they do not contain any symbols. 
body mass index

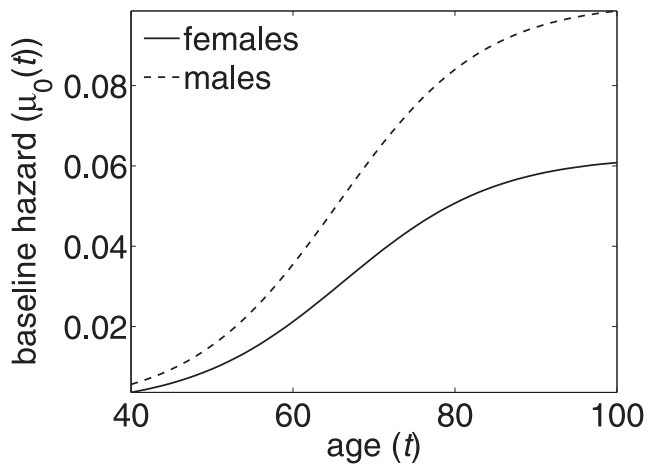

hematocrit

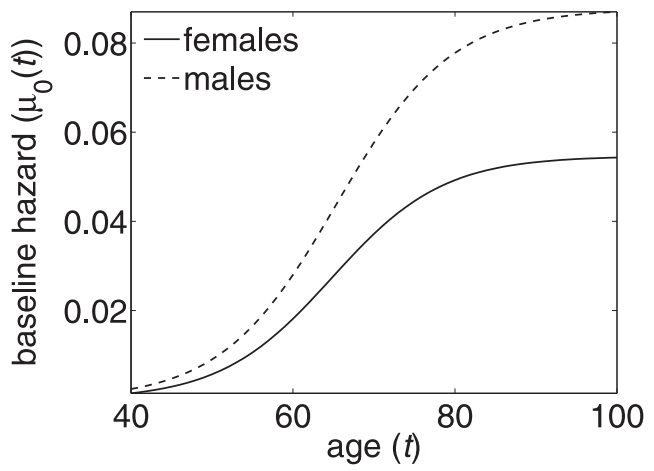

pulse rate

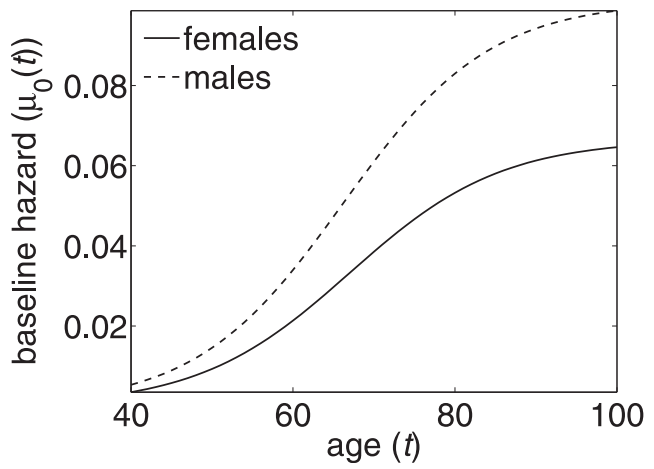

diastolic blood pressure

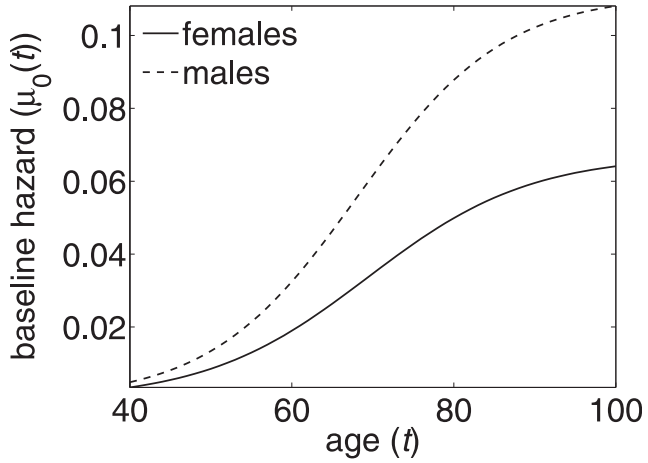

pulse pressure

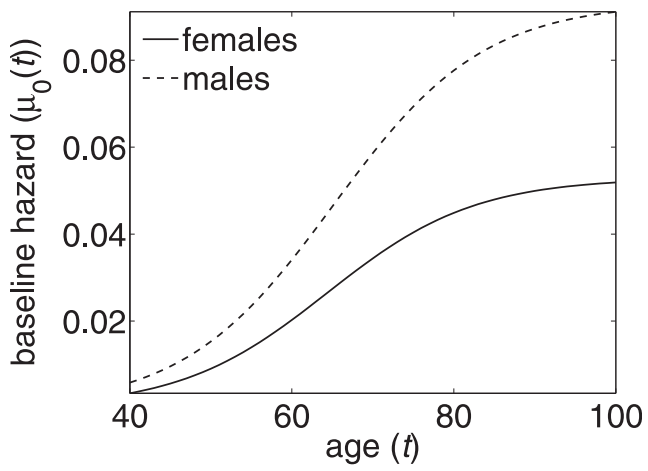

serum cholesterol

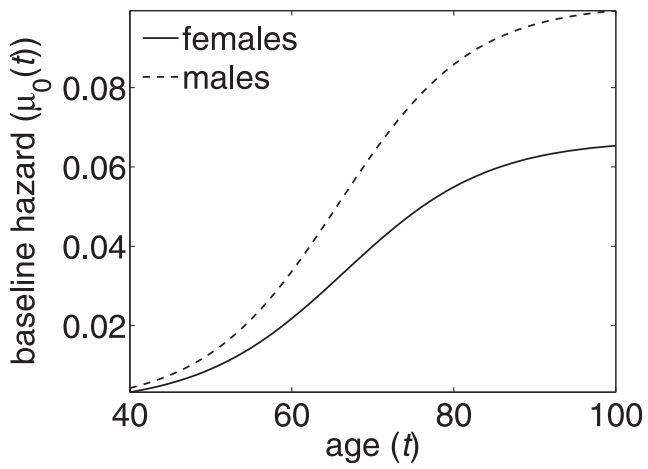

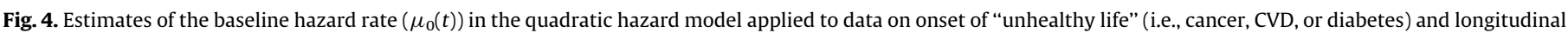

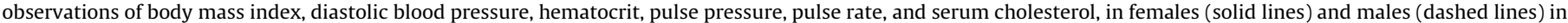
the Framingham Heart Study (original cohort).

and the "price" for the same magnitude of deviation from the "norm" (in terms of an absolute increase in the risk of onset of "unhealthy life" compared to the baseline level $\mu_{0}(t)$ at that age) is higher at older ages. For all indices (except for hematocrit), the trajectory of "vulnerability" index for males lies above that of females meaning the higher "price" for deviations from the norm for males compared to females (corresponding to a narrower Ushape of the risk at each age in males). This suggests that males have generally lower resistance to stresses than females: males are more vulnerable to deviations from the "normal" state caused by external disturbances (the same magnitude of deviation from the "optimal" trajectory results in a larger increase in the risk compared to females). In addition, the trajectories of $Q(t)$ increase faster in males than in females for all indices except for hematocrit. This means the faster decline in resistance to stresses in males compared to females. Combined with higher values of the baseline hazard, this contributes to the observed higher incidence rate for males, as well as to its faster increase with age (Fig. 2).

\subsection{Decline in adaptive capacity}

The absolute value of the feedback coefficient $a(t)$ tends to decline with age in both females and males, see Fig. 6 (the decline is significant at different levels in all cases except for HCT in males, see Table 1 ). This means that the adaptive capacity declines with age in both females and males for all analysed physiological indices and more time is needed for the trajectory of an index $\left(Y_{t}\right)$ to approach the "mean allostatic state" $\left(f_{1}(t)\right)$ at older ages compared to younger ages. The rate of decline differs in females and males for different indices, as well as the initial values at age 40 . For example, both the rate of decline and the (absolute value of) adaptive capacity at age 40 are higher in males than in females for DBP, while the opposite is observed for HCT and PP. In these cases, the trajectories for females and males intersect at ages about 60-70. For PP and $\mathrm{SCH}$, the trajectories for females and males go almost in parallel, but the relationship between the values of adaptive capacity at age 40 is the opposite for these two indices. These 

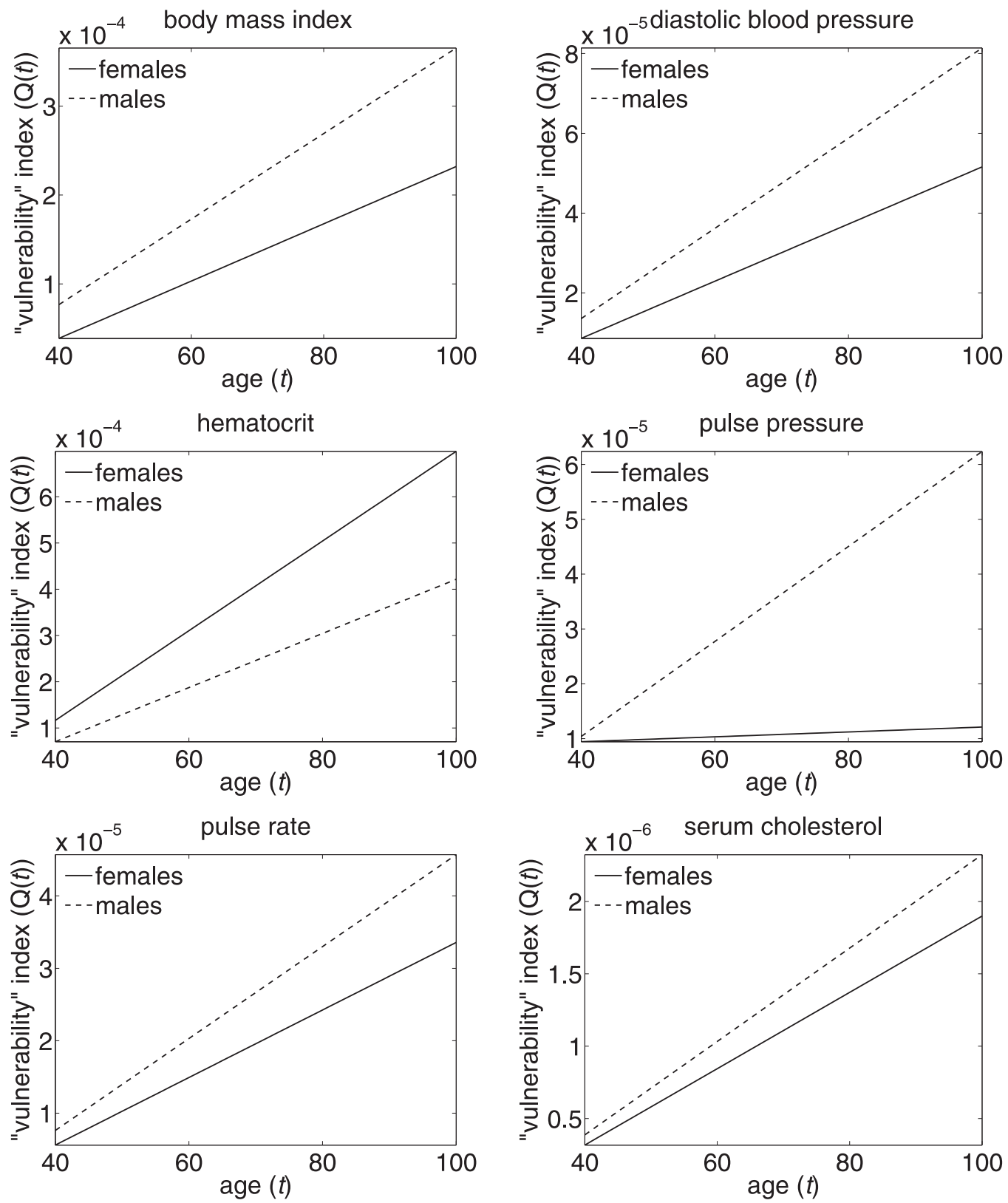

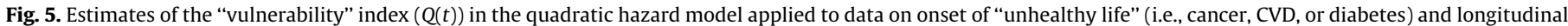

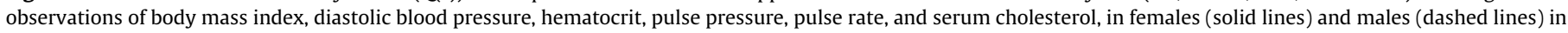
the Framingham Heart Study (original cohort).

observations indicate that the mechanisms underlying the decline in the adaptive capacity in females and males may not work universally for all physiological indices and deserve further study.

\subsection{Allostatic adaptation and "Optimal" trajectories}

For all analysed indices and both sexes, the resulting estimates of the "mean allostatic state" $f_{1}(t)$ are age-dependent ( $p$-values for the null hypothesis about the constant $f_{1}(t)$ are: $p=0.0002$ for PR in males, $p<0.0001$ for all other cases). The "optimal" trajectories $f(t)$ are also age-dependent, however, the null hypothesis about the constant $f(t)$ was rejected only for BMI $(p=0.035)$ and DBP $(p=0.0003)$ in females and HCT $(p=0.035)$ in males. Agedependence of the "optimal" trajectory $f(t)$ indicates that the values of these indices that minimise the risk of onset of unhealthy life for young and middle-age adults may differ from those of old and oldest-old individuals. Therefore, the strategies aiming at maintaining the level of a physiological index at oldest ages similar to the level optimal for younger individuals may actually increase the risk of development of cancer, CVD or diabetes (Yashin et al., 2009). Furthermore, the notion of the "norm" used here corresponds, by definition, to the minimal risk of onset of any of the three diseases (cancer, CVD, diabetes) at respective ages. Hence, it is a "compromise" measure which may deviate from the disease-specific "norms" (the latter may have different patterns if a physiological index has non-uniform impact on the different disease risks). However, if the goal is to minimise the risk of onset of any of the three diseases rather than to minimise the risk of one disease at a possible cost of increased risks for other diseases, then such approach may be preferable to analyses of disease-specific data. Also, it may happen that the trajectory that minimises the risk of onset of "unhealthy life" differs somewhat from that minimising 

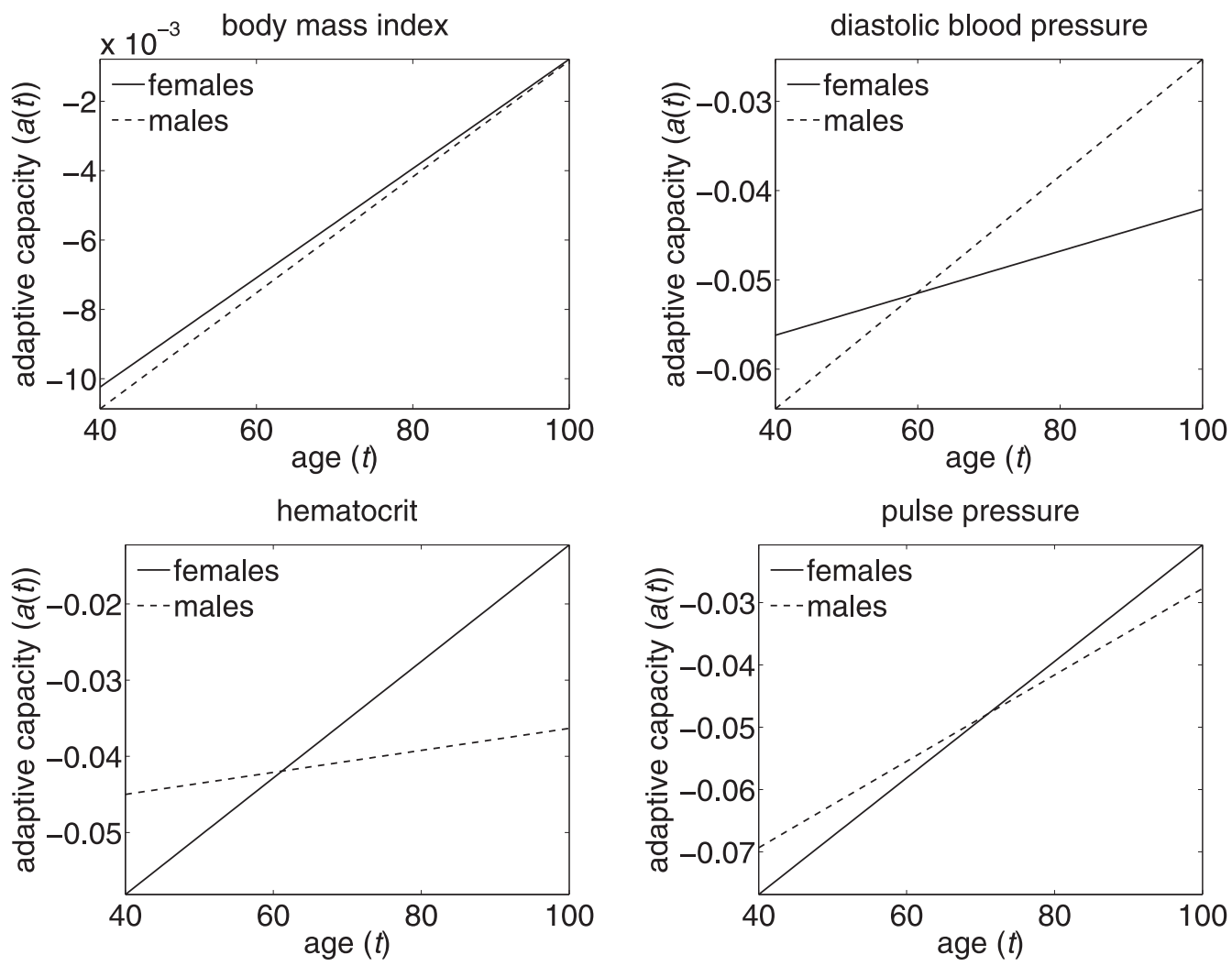

pulse pressure

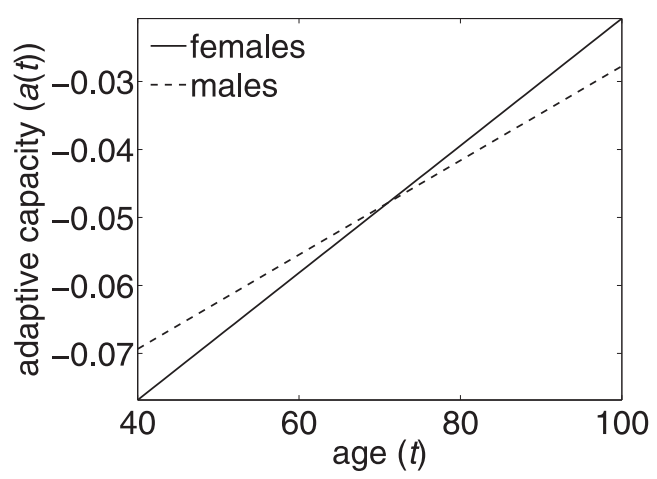

pulse rate
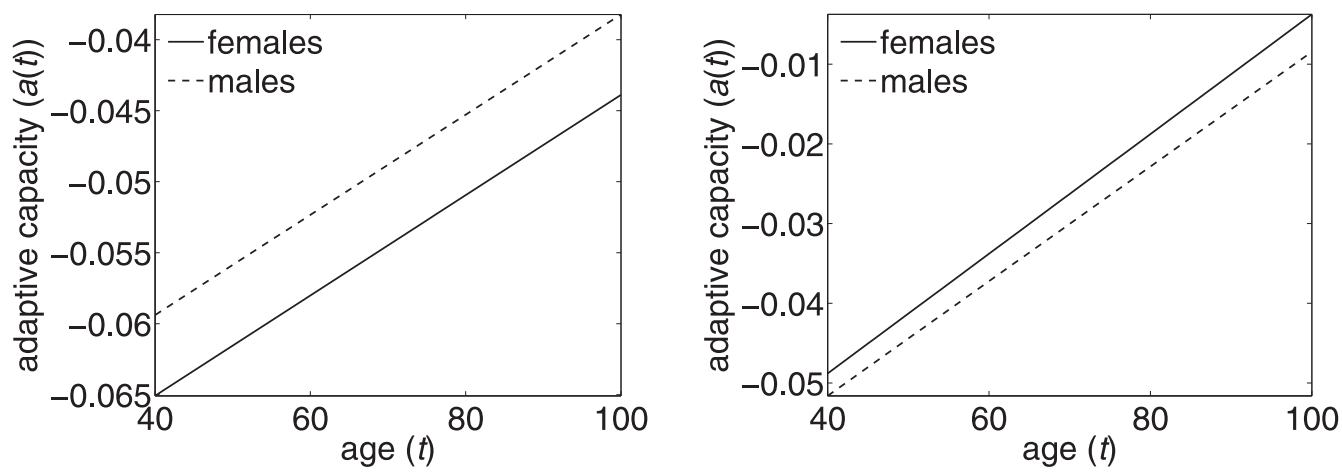

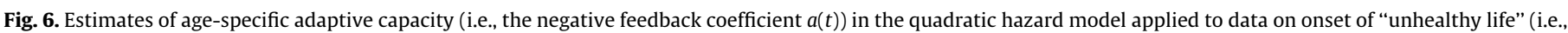

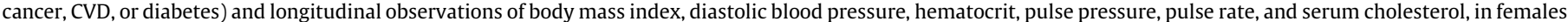
(solid lines) and males (dashed lines) in the Framingham Heart Study (original cohort).

the risk of death. For example, at old ages, when the risk of a disease may decline, total mortality risk continues to increase, so that the factors minimising the risks of the disease and death may be different (Ukraintseva and Yashin, 2003). Thus, the concept of physiological "norm" deserves additional studies, which need to be performed to evaluate the "optimal" physiological trajectories corresponding to minimal mortality risks at different ages and to compare them with those trajectories evaluated here for the onset of "unhealthy life."

\subsection{Measures of allostatic load and differences in healthy life expectancy}

For all indices and both sexes, the null hypotheses on coincidence of $f_{1}(t)$ and $f(t)$ (in the model with quadratic $f_{1}(t)$ and $f(t))$ were rejected $(0.01 \leq p<0.05$ for HCT and SCH in females, $p=0.008$ for BMI in males, $p<0.0001$ in other cases). This means that the processes of compensatory adaptation and remodelling regulating the age dynamics of respective physiological states force the age trajectories of the indices to follow the curves which do not tend to minimise the risk of onset of "unhealthy life." Persistent deviations from the "norm" characterise the effects of allostatic adaptation and the magnitudes of such deviations for each physiological index can be associated with components of allostatic load leading to increased chances of development of the diseases (if the estimates of the quadratic term in the hazard, $Q(t)$, are not zero, which is the case in our applications). If one takes two individuals, the first one being a "typical person" whose age trajectory of some physiological index drifts along the "mean allostatic state" $f_{1}(t)$ and the second one who manages to keep his/ her age trajectory at the "optimal" level corresponding to $f(t)$, then the first individual will have increased chances of getting the diseases compared to the second one (if all other factors, i.e., those summarised in $\mu_{0}(t)$, are the same). As the result, the "healthy life expectancy" in two individuals will be different and such a difference can be evaluated from the model. In case of our 
applications, the difference in healthy life expectancy reaches 6.7 years for PP in females and 3.0 years for PP in males, although for other indices the increase is more moderate (about $1-2$ years) indicating that the impact of other (unobserved) factors (summarised collectively by $\mu_{0}(\mathrm{t})$ may be more pronounced in defining healthy lifespan compared to the analysed indices.

\subsection{Limitations of the study and alternative approaches}

The limitations of the approach considered in this paper deal with the fact that it evaluates average effects of allostatic adaptation and average "optimal" functions. It would be useful to investigate individual dynamic effects of allostatic adaptation and individual optimal age trajectories of physiological indices. Such extension, however, would involve more data and require development of more sophisticated dynamic models describing numerous aging related changes in their mutual connection and their effects on mortality/morbidity risk. One more limitation is that the approach uses a one-dimensional description of physiological state. However, different physiological indices may be mutually dependent and evaluating the "norms" and other parameters independently may introduce a bias in estimates. Thus, multidimensional analysis of different indices is important. At the same time, a substantial deviation of some indices from their one-dimensional "norms" may serve as an important indicator of a possible pathological development that may lead to overt symptoms of chronic diseases.

The method used in this paper for modelling aging related changes is not the only option to analyse health deterioration with age or relationship between longitudinal measurements and mortality/morbidity data. Different methods for joint modelling of longitudinal and time-to-event data have been developed in biostatistics (see, e.g., reviews in Tsiatis and Davidian, 2004; Yu et al., 2004). The reliability literature presents approaches for analyses of failure time-degradation data with covariates which provide a powerful tool for investigation of effects of internal (degradation, wear) and external (covariates, stresses) explanatory variables on failure time distributions (see recent papers by Bagdonavicius and Nikulin, 2009; Lehmann, 2009). Other approaches can be applied in different circumstances depending on availability of additional information apart from data on incidence of diseases. For example, the (random) vitality or a firstpassage type models (Arbeev et al., 2005; Finkelstein, 2007, 2009; $\mathrm{Li}$ and Anderson, 2009; Strehler and Mildvan, 1960) can be employed to investigate the shapes of the incidence rate curves. The availability of individual data on minor health deficits and symptoms collected in surveys allows for construction of a cumulative measure of health deterioration called a frailty index or an index of cumulative deficits (Goggins et al., 2005; Kulminski et al., 2007, 2008b, 2008c; Mitnitski et al., 2001; Rockwood and Mitnitski, 2007; Yashin et al., 2007b,c) which proved to be an efficient empirical approach to investigate aging related processes of health deterioration. The availability of individual measurements of biological parameters reflecting functioning of the hypothalamic-pituitary-adrenal axis, sympathetic nervous system, cardiovascular system, and metabolic processes allows for calculation of cumulative physiological wear and tear measure of allostatic load and its effect on health outcomes (Seeman et al., 1997, 2001). However, information on individual measurements of relevant biomarkers (such as those characterising stress resistance, adaptive capacity, allostatic load, etc.) is often not available and some indirect methods are needed to investigate the impact of respective aging-related processes on mortality/morbidity risks. The approach by Yashin et al. (2007a) provides a powerful tool for comprehensive analyses of longitudinal data on aging, health, and longevity that allows for incorporating such aging-related mechanisms into the model and evaluating them from data on physiological indices and mortality/morbidity. This approach can quantify important connection between aging and development of chronic conditions, which have never been evaluated from the analyses of longitudinal data before.

\section{Conclusions}

The results of this study show that the model of human mortality and aging (Yashin et al., 2007a) can be successfully used for investigating physiological mechanisms regulating duration of healthy life. The analyses demonstrate how decline in resistance to stresses and adaptive capacity, accompanying human aging, can be evaluated from longitudinal data and show how these key components of the aging process contribute to an increase in the risk of onset of unhealthy life. The results indicate the presence of substantial gender difference in aging related decline in stress resistance and adaptive capacity, as well as in accumulation of allostatic load, which contributes to the difference in the shape of the sex-specific patterns of incidence rates of aging related diseases. These differences may have genetic origin or may be caused by different roles males and females play in society. The determinants of such differences deserve further study.

\section{Acknowledgements}

The research reported in this paper was supported by the National Institute on Aging grants R01AG028259, R01AG030198 and R01AG027019. The content is solely the responsibility of the authors and does not necessarily represent the official views of the National Institute on Aging or the National Institutes of Health. The Framingham Heart Study (FHS) is conducted and supported by the NHLBI in collaboration with the FHS Investigators. This manuscript was prepared using a limited access dataset obtained from the NHLBI and does not necessarily reflect the opinions or views of the FHS or the NHLBI.

\section{Appendix A}

\section{A.1. Sensitivity analyses}

\section{A.1.1. Shape of the "allostatic trajectory" $\left(f_{1}(t)\right)$}

The "basic" model applied to data on onset of "unhealthy life" used a quadratic function to specify the "allostatic trajectory" $f_{1}(t)$. We also estimated the model with linear $f_{1}(t)$ and tested the null hypothesis in favour of the linear model. The null hypothesis was rejected for all indices and both sexes $(p<0.0001$ in all cases except pulse rate for males $(p=0.002)$ ) excluding pulse pressure for females $(p=0.1)$. The model with linear $f_{1}(t)$ applied to for data on pulse pressure for females produced the estimates similar to the model with quadratic $f_{1}(t)$ shown in Table 1 .

\section{A.1.2. Non-symmetric "vulnerability" index $(Q(t))$}

The "basic" model described in the text of the manuscript assumes the symmetric increase in the risk due to the same magnitude of deviations of a physiological index to the left (i.e., smaller than "optimal" values) or to the right (i.e., larger than "optimal" values) from the "optimal" trajectory $f(t)$. We also estimated the model assuming a non-symmetric form of $Q(t)$ (i.e., different "penalties," in terms of an additional risk, for deviations of the index to the left and to the right from the "optimal" trajectory $f(t)$ ). Respective terms are modelled by two linear functions, $Q_{L}(t)$ and $Q_{R}(t)$. We tested the respective hypotheses on whether the "penalties" for deviations of the index to 
the left and to the right from the "norm" $f(t)$ are different. In all cases except for diastolic blood pressure in males $(p=0.037)$ the respective null hypotheses in favour of the simpler model (i.e., symmetric "penalties" for deviations to the left and to the right from the "norm," as used in the "basic" model) were not rejected at the 0.05 significance level. The model with non-symmetric vulnerability index applied to data on diastolic blood pressure in males estimated a steeper increase of $Q_{L}(t)$ than $Q_{R}(t)$ and a lower "optimal" trajectory $\left(a_{f}=69.5\right.$, $\left.b_{f}=-0.051\right)$ than the original model, with estimates of all other parameters close to that in the "symmetric" model shown in Table 1.

\section{A.1.3. Shape of the "optimal" trajectory $(f(t))$}

The "basic" model used a linear approximation of the "optimal" trajectory $f(t)$. We also estimated the model with quadratic $f(t)$ and tested the null hypothesis in favour of the linear model. The null hypothesis was not rejected at the 0.05 significance level for all indices and both sexes except pulse pressure for males $(p=0.008)$ and pulse rate for females $(p=0.007)$. The model with quadratic $f(t)$ applied to data on pulse pressure for males and pulse rate for females produced the shapes of all components (except $f(t)$ ) similar to those in the model with linear $f(t)$ shown in Table 1 . We also tested the null hypothesis that "optimal" values do not change with age in the model with quadratic $f(t)$. The null hypothesis was rejected at the 0.05 significance level for pulse rate in females $(p=0.022)$ and pulse pressure in males $(p=0.008)$

\section{A.1.4. Optimal ranges of values of physiological indices}

The "basic" model assumes a single "optimal" value of a physiological index that corresponds to the minimal risk of onset of "unhealthy life" at respective age. Alternatively, we can assume that there is a range of such values minimising the risk at each age. To check such scenario, we estimated the model with "optimal range," or

$$
\begin{aligned}
\mu\left(t, Y_{t}\right)= & \mu_{0}(t)+\left(Y_{t}-f_{L}(t)\right)^{2} I\left(Y_{t}<f_{L}(t)\right) Q_{L}(t) \\
& +\left(Y_{t}-f_{R}(t)\right)^{2} I\left(Y_{t}>f_{L}(t)\right) Q_{R}(t)
\end{aligned}
$$

where $I($.$) is an indicator function (which equals one if the$ condition in the parentheses holds and zero otherwise), $f_{L}(t)$ and $f_{R}(t)$ are two functions defining the left and right boundaries of the "optimal range," which were modelled as linear functions.

We tested the respective null hypotheses in favour of the simpler model (i.e., with a single "optimal" value) and the null hypotheses were not rejected at the 0.05 level in all cases. Nevertheless, the optimal ranges $f_{L}(t)$ and $f_{R}(t)$, rather than the optimal trajectories $f(t)$, were estimated for the majority of indices. In all cases where such ranges were estimated, the ranges narrowed with age indicating that the range of "acceptable" values of indices with minimal risk of onset of "unhealthy life" shrinks as an individual gets older. That is, the range of values of a physiological index with the same absolute increase in the risk (compared to the baseline level at respective age) narrows with age which can be considered as a manifestation of decline in stress resistance with age. This leads to increased chances of development of the diseases at older ages, compared to younger ages, associated with the same magnitude of deviation from the "optimal" level.

\section{References}

Allison, D.B., Faith, M.S., Heo, M., Kotler, D.P., 1997. Hypothesis concerning the Ushaped relation between body mass index and mortality. Am. J. Epidemiol. 146 (4), 339-349.

Arbeev, K.G., Ukraintseva, S.V., Arbeeva, L.S., Yashin, A.I., 2005. Mathematical models for human cancer incidence rates. Demographic Res. 12 (10), 237-271.
Arbeev, K.G., Akushevich, I., Kulminski, A.M., Arbeeva, L.S., Akushevich, L., Ukraintseva, S.V Culminskaya, IV Yashin, A.I. 2009. Genetic model for longitudinal studies of aging, health, and longevity and its potential application to incomplete data. J. Theor. Biol. 258 (1), 103-111.

Bagdonavicius, V., Nikulin, M., 2009. Statistical models to analyse failure, wear, fatigue, and degradation data with explanatory variables. Communications in Statistics-Theory and Methods, 38pp. 3031-3047 16-17.

Boutitie, F., Gueyffier, F., Pocock, S., Fagard, R., Boissel, J.P., 2002. J-shaped relationship between blood pressure and mortality in hypertensive patients: new insights from a meta-analysis of individual-patient data. Ann. Intern. Med. 136 (6), 438-448.

Dawber, T.R., 1980. The Framingham Study: The Epidemiology of Atherosclerotic Disease. Harvard University Press, Cambridge, MA.

Dawber, T.R., Meadors, G.F., Moore, F.E., 1951. Epidemiological approaches to heart disease: the Framingham study. Am. J. Public Health 41 (3), 279-286.

Finkelstein, M., 2007. Aging: damage accumulation versus increasing mortality rate. Math. Biosci. 207 (1), 104-112.

Finkelstein, M., 2009. On damage accumulation and biological aging. J. Stat. Plan.Inf. 139 (5), 1643-1648.

Goggins, W.B., Woo, J., Sham, A., Ho, S.C., 2005. Frailty index as a measure of biological age in a Chinese population. J. Gerontol. A. Biol. Sci. Med. Sci. 60 (8), 1046-1051.

Hall, D.M., Xu, L., Drake, V.J., Oberley, L.W., Oberley, T.D., Moseley, P.L., Kregel, K.C. 2000. Aging reduces adaptive capacity and stress protein expression in the liver after heat stress. J. Appl. Physiol. 89 (2), 749-759.

Kulminski, A., Ukraintseva, S.V., Akushevich, I., Arbeev, K.G., Land, K., Yashin, A.I., 2007. Accelerated accumulation of health deficits as a characteristic of aging. Exp. Gerontol. 42 (10), 963-970.

Kulminski, A.M., Arbeev, K.G., Kulminskaya, I.V., Ukraintseva, S.V., Land, K., Akushevich, I., Yashin, A.I., 2008a. Body mass index and nine-year mortality in disabled and nondisabled older U.S. Individuals. J. Am. Geriatr. Soc. 56 (1), 105-110.

Kulminski, A.M., Arbeev, K.G., Ukraintseva, S.V., Culminskaya, I.V., Land, K., Yashin, A.I., 2008b. Changes in health status among participants of the Framingham heart study from the 1960s to the 1990s: application of an index of cumulative deficits. Ann. Epidemiol. 18 (9), 696-701.

Kulminski, A.M., Ukraintseva, S.V., Culminskaya, I.V., Arbeev, K.G., Land, K.C. Akushevich, L., Yashin, A.I., 2008c. Cumulative deficits and physiological indices as predictors of mortality and long life. J. Gerontol. A. Biol. Sci. Med. Sci. 63 (10), 1053-1059.

Kuzuya, M., Enoki, H., Iwata, M., Hasegawa, J., Hirakawa, Y., 2008. J-shaped relationship between resting pulse rate and all-cause mortality in communitydwelling older people with disabilities. J. Am. Geriatr. Soc. 56 (2), 367-368.

Lehmann, A., 2009. Joint modelling of degradation and failure time data. J. StatPlan.Inf. 139 (5), 1693-1706.

Li, T., Anderson, J.J., 2009. The vitality model: a way to understand population survival and demographic heterogeneity. Theor. Popul. Biol. 76 (2), 118-131.

Mazza, A., Zamboni, S., Rizzato, E., Pessina, A.C., Tikhonoff, V., Schiavon, L., Casiglia, E., 2007. Serum uric acid shows a J-shaped trend with coronary mortality in non-insulin-dependent diabetic elderly people the Cardiovascular Study in the Elderly (CASTEL). Acta Diabetol. 44 (3), 99-105.

McEwen, B.S., 2000. Allostasis and allostatic load: implications for neuropsychopharmacology. Neuropsychopharmacology 22 (2), 108-124.

McEwen, B.S., Wingfield, J.C., 2003. The concept of allostasis in biology and biomedicine. Horm. Behav. 43 (1), 2-15.

Mitnitski, A.B., Mogilner, A.J., Rockwood, K., 2001. Accumulation of deficits as a proxy measure of aging. Scientific World Journal 1, 323-336.

Okumiya, K., Matsubayashi, K., Wada, T., Fujisawa, M., Osaki, Y., Doi, Y., Yasuda, N., Ozawa, T., 1999. A U-shaped association between home systolic blood pressure and four-year mortality in community-dwelling older men. J. Am. Geriatr. Soc. 47 (12), 1415-1421.

Protogerou, A.D., Safar, M.E., Iaria, P., Safar, H., Le Dudal, K., Filipovsky, J., Henry, O., Ducimetiere, P., Blacher, J., 2007. Diastolic blood pressure and mortality in the elderly with cardiovascular disease. Hypertension 50 (1), 172-180.

Rankin, M.M., Kushner, J.A., 2009. Adaptive beta-cell proliferation is severely restricted with advanced age. Diabetes 58 (6), 1365-1372.

Rockwood, K., Mitnitski, A., 2007. Frailty in relation to the accumulation of deficits. J. Gerontol. A. Biol. Sci. Med. Sci. 62 (7), 722-727.

Rockwood, K., Awalt, E., MacKnight, C., McDowell, I., 2000. Incidence and outcomes of diabetes mellitus in elderly people: report from the canadian study of health and aging. Can. Med. Assoc. J. 162 (6), 769-772.

Seeman, T.E. Singer, B.H., Rowe, J.W., Horwitz, R.I., McEwen, B.S., 1997. Price of adaptation-Allostatic load and its health consequences: MacArthur studies of successful aging. Arch. Intern. Med. 157 (19), 2259-2268.

Seeman, T.E., McEwen, B.S., Rowe, J.W., Singer, B.H., 2001. Allostatic load as a marker of cumulative biological risk: MacArthur studies of successful aging. Proc. Natl. Acad. Sci. U.S.A. 98 (8), 4770-4775.

Sterling, P., Eyer, J., 1988. Allostasis: a new paradigm to explain arousal pathology. In: Fisher, S., Reason, J. (Eds.), Handbook of Life Stress, Cognition and Health. John Wiley \& Sons, New York, pp. 629-649.

Strehler, B., 1962. Time, Cells, and Aging. Academic Press, London.

Strehler, B.L., Mildvan, A.S., 1960. General theory of mortality and aging. Science 132 (3418), 14-21.

Troiano, R.P., Frongillo, E.A., Sobal, J., Levitsky, D.A., 1996. The relationship between body weight and mortality: A quantitative analysis of combined information from existing studies. Int. J. Obesity 20 (1), 63-75. 
Troncale, J.A., 1996. The aging process: physiologic changes and pharmacologic implications. Postgrad. Med. 99 (5), 111-114 120-122.

Tsiatis, A.A., Davidian, M., 2004. Joint modelling of longitudinal and time-to-event data: an overview. Statistica Sinica 14 (3), 809-834.

Ukraintseva, S.V., Yashin, A.I., 2001. How individual age-associated changes may influence human morbidity and mortality patterns. Mech. Ageing Dev. 122 (13), $1447-1460$.

Ukraintseva, S.V., Yashin, A.I., 2003. Individual aging and cancer risk: How are they related? Demographic Res. 9 (8), 163-196.

Ukraintseva, S., Sloan, F., Arbeev, K., Yashin, A., 2006. Increasing rates of dementia at time of declining mortality from stroke. Stroke 37 (5), 1155-1159.

Ukraintseva, S.V., Arbeev, K.G., Akushevich, I., Kulminski, A., Arbeeva, L., Culminskaya, I., Akushevich, L., Yashin, A.I., 2010. Trade-offs between cancer and other diseases: Do they exist and influence longevity? Rejuvenation Research 13 (4), 387-396.

van Uffelen, J.G.Z., Berecki-Gisolf, J., Brown, W.J., Dobson, A.J., 2010. What is a healthy body mass index for women in their seventies? Results from the australian longitudinal study on women's health. J. Gerontol. A: Biol. Sci. Med. Sci. 65 (8), 844-850.

Witteman, J.C.M., Grobbee, D.E., Valkenburg, H.A., Vanhemert, A.M., Stijnen, T., Burger H., Hofman, A., 1994. J-shaped relation between change in diastolic bloodpressure and progression of aortic atherosclerosis. Lancet 343 (8896), 504-507.

Yashin, A.I., Ukraintseva, S.V., De Benedictis, G., Anisimov, V.N., Butov, A.A., Arbeev, K., Jdanov, D.A., Boiko, S.I., Begun, A.S., Bonafe, M., Franceschi, C., 2001. Have the oldest old adults ever been frail in the past? A hypothesis that explains modern trends in survival. J. Gerontol. A: Biol. Sci. Med. Sci. 56 (10), B432-B442.

Yashin, A.I., Akushevich, I.V., Arbeev, K.G., Akushevich, L., Ukraintseva, S.V., Kulminski, A., 2006. Insights on aging and exceptional longevity from longitudinal data: novel findings from the Framingham heart study. Age 28 (4), 363-374.
Yashin, A.I., Arbeev, K.G., Akushevich, I., Kulminski, A., Akushevich, L., Ukraintseva, S.V., 2007a. Stochastic model for analysis of longitudinal data on aging and mortality. Math. Biosci. 208 (2), 538-551.

Yashin, A.I., Arbeev, K.G., Kulminski, A., Akushevich, I., Akushevich, L., Ukraintseva, S.V., 2007b. Cumulative index of elderly disorders and its dynamic contribution to mortality and longevity. Rejuvenation Res. 10 (1), 75-86.

Yashin, A.I., Arbeev, K.G., Kulminski, A., Akushevich, I., Akushevich, L., Ukraintseva, S.V., 2007c. Health decline, aging and mortality: how are they related? Biogerontology 8 (3), 291-302.

Yashin, A.I., Arbeev, K.G., Kulminski, A., Akushevich, I., Akushevich, L., Ukraintseva, S.V., 2008. What age trajectories of cumulative deficits and medical costs tell us about individual aging and mortality risk: findings from the NLTCS-Medicare data. Mech. Ageing Dev. 129 (4), 191-200.

Yashin, A.I., Ukraintseva, S.V., Arbeev, K.G., Akushevich, I., Arbeeva, L.S., Kulminski, A.M., 2009. Maintaining physiological state for exceptional survival: What is the normal level of blood glucose and does it change with age? Mech. Ageing Dev. 130 (9), 611-618.

Yashin, A.I., Arbeev, K.G., Akushevich, I., Arbeeva, L., Kravchenko, J., Il'yasova, D., Kulminski, A., Akushevich, L., Culminskaya, I., Wu, D., Ukraintseva, S.V., 2010a. Dynamic determinants of longevity and exceptional health. Curr.Gerontol.Geriatr.Res. Article ID 381637.

Yashin, A.I., Arbeev, K.G., Akushevich, I., Ukraintseva, S.V., Kulminski, A., Arbeeva, L.S., Culminskaya, I., 2010b. Exceptional survivors have lower age trajectories of blood glucose: lessons from longitudinal data. Biogerontology 11 (3), 257 265.

Yu, M.G., Law, N.J., Taylor, J.M.G., Sandler, H.M., 2004. Joint longitudinal-survivalcure models and their application to prostate cancer. Statistica Sinica 14 (3) 835-862. 